\section{Paclitaxel bei NSCLC angepasst dosieren}

\begin{abstract}
In dieser Studie wurde untersucht, ob eine pharmakokinetisch kontrollierte Paclitaxel-Dosierung bei Patienten mit fortgeschrittenem nichtkleinzelligem Lungenkarzinom (NSCLC) die Toxizitäten reduzieren kann.
\end{abstract}

$\mathrm{P}$ aclitaxel ist ein Standard beim NSCLC. Allerdings können Toxizitäten wie Neutropenie und periphere Neuropathie die Lebensqualität beeinträchtigen. Daher könnte die Pharmakokinetik bedeutsam sein. Sie schwankt interindividuell stark, gleichzeitig besteht ein Zusammenhang zwischen Exposition und Toxizität. In einer Phase-III-Studie wurde nun eine pharmakokinetisch kontrollierte Paclitaxel-Dosierung mit dem Ziel untersucht, eine Exposition über oder unterhalb der Therapieschwelle zu vermeiden. 365 Patienten mit neu diagnostiziertem Tumor erhielten randomisiert bis zu 6 3-Wochen-Zyklen Carboplatin (AUC 6) oder Cisplatin $\left(80 \mathrm{mg} / \mathrm{m}^{2}\right)$ in Kombination mit Paclitaxel entweder in der Standarddosierung von $200 \mathrm{mg} /$ $\mathrm{m}^{2}$ (Arm A) oder in pharmakokinetisch kontrollierter Dosierung (Arm B). Dabei wurde die initiale Paclitaxel-Dosis nach Körperoberfläche, Alter und Geschlecht berechnet. Nachfolgende Dosierungen wurden an das Auftreten einer Neutropenie und die Paclitaxel-Exposition in den vorhergehenden Zyklen angepasst.

Nach median 21,6 Monaten waren Neutropenien vom Grad 4 (primärer Endpunkt) in beiden Armen gleich häufig (19 vs. $16 \%$; $p=0,10)$, Neuropathien vom $\operatorname{Grad} \geq 2$ ( 38 vs. $23 \%$; $p<0,001$ ) oder $\geq 3$ ( 9 vs. $2 \%$; $p<0,001$ ) in Arm B signifikant seltener. Die mediane PaclitaxelEnddosis war in Arm B signifikant niedriger als bei Standarddosierung (199 vs. $\left.150 \mathrm{mg} / \mathrm{m}^{2} ; \mathrm{p}<0,001\right)$. Der Anteil an Patienten mit einer Paclitaxel-Exposition über der therapeutischen Schwelle sank von $38 \%$ in Zyklus 1 auf $2 \%$ in Zyklus 6.
Das Ansprechen war in beiden Armen vergleichbar (31 vs. $27 \%$; $=0,405$ ), ebenso adjustiertes medianes progressionsfreies Überleben (5,5 vs. 4,9 Monate; Hazard Ratio [HR] 1,16; $\mathrm{p}=0,228)$ und Gesamtüberleben (10,1 vs. 9,5 Monate; HR 1,$05 ; \mathrm{p}=0,682$ ).

Fazit: Die pharmakokinetisch kontrollierte Dosierung von Paclitaxel führte nicht zur Reduktion schwerer Neutropenien, aber zur Abnahme therapieassoziierter Neuropathien. Somit verbesserte dieses Vorgehen das Nutzen-Risiko-Profil von Paclitaxel bei Patienten mit fortgeschrittenem NSCLC. Das könnte besonders wertvoll sein, wenn die Patienten gebrechlich sind, extremes Körpergewicht, Leberfunktionsstörungen und ein hohes Risiko für therapieinduzierte Neuropathien haben. Judith Neumaier

Joerger M et al. Open-label, randomized study of individualized, pharmacokinetically

(PK)-guided dosing of paclitaxel combined with carboplatin or cisplatin in patients with advanced non-small-cell lung cancer (NSCLC). Ann Oncol. 2016;27(10):1895-902.

\title{
NSCLC: Drittgenerations-Anthrazyklin geprüft
}

\section{Amrubicin ist zur Behandlung des nichtkleinzelligen Lungenkarzinoms und des kleinzelligen Lungenkarzi- noms zugelassen. Nun wurde der Wirkstoff bei Patienten mit vorbe- handeltem NSCLC mit Docetaxel verglichen.}

A mrubicin, ein Anthrazyklin der dritten Generation, ist ein potenter Inhibitor der Topoisomerase II. In bisherigen Phase-II-Studien ohne Kontrollgruppe wurden unter Amrubicin ein medianes progressionsfreies Überleben von 3,3 Monaten und ein medianes Gesamtüberleben von 12 Monaten erreicht. Nun wurde in einer randomisierten Phase-III-Studie geprüft, ob Amrubicin gegenüber Docetaxel hinsichtlich des progressionsfreien Überlebens überlegen ist.

202 japanische Patienten mit einem nichtkleinzelligen Lungenkarzinom
(NSCLC), die mit 1-2 Chemotherapien vorbehandelt waren, nahmen an der Studie teil. Die Patienten erhielten randomisiert entweder Amrubicin (35 mg/ $\mathrm{m}^{2}$ an den Tagen 1-3 alle 3 Wochen) oder Docetaxel $\left(60 \mathrm{mg} / \mathrm{m}^{2}\right.$ an Tag 1 alle 3 Wochen).

Das mediane progressionsfreie Überleben (primärer Endpunkt: 3,6 vs. 3,0 Monate; Hazard Ratio [HR] 0,90; $\mathrm{p}=0,54)$ und das Gesamtüberleben $(14,6$ vs. 13,5 Monate; $\operatorname{HR} 0,97 ; \mathrm{p}=0,86$ ) waren in beiden Gruppen vergleichbar. Auch in den Subgruppen gab es hinsichtlich des progressionsfreien Überlebens und des Gesamtüberlebens keinen signifikanten Unterschied zwischen den beiden Therapien. Die Gesamtansprechrate betrug unter Amrubicin 14,4\% und unter Docetaxel 19,6\% ( $p=0,45)$. Bei $55,7 \%$ der Patienten wurde Krankheitskontrolle erreicht.

Unerwünschte Ereignisse traten in beiden Armen bei allen Patienten auf.
Eine Neutropenie vom Grad $\geq 3$ war bei 82,7 bzw. 78,8\% der Patienten zu beobachten, eine Leukopenie vom Grad $\geq 3$ bei 63,3 bzw. $70,7 \%$ und eine febrile Neutropenie vom Grad $\geq 3$ bei 13,3 bzw. $18,2 \% .3$ von 8 unerwünschten kardialen Ereignissen in der Amrubicin-Gruppe wurden mit dieser Therapie in Zusammenhang gebracht. Die kardialen Nebenwirkungen verschwanden nach Beendigung der Therapie wieder.

Fazit: Dies ist die erste Phase-III-Studie, in der Amrubicin und Docetaxel bei vorbehandelten NSCLC-Patienten direkt miteinander verglichen wurden. Dabei zeigte Amrubicin keine Überlegenheit gegenüber Docetaxel. Das Anthrazyklin der dritten Generation verbesserte das progressionsfreie Überleben nicht signifikant im Vergleich zu Docetaxel.

Judith Neumaier

Yoshioka $\mathrm{H}$ et al. A randomized, open-label, phase III trial comparing amrubicin versus docetaxel in patients with previously treated non-small-cell lung cancer. Ann Oncol. 2017; 28(2):285-291. 